\title{
Primary Hodgkin lymphoma of the lung arising with hemoptysis and pulmonary consolidation: a case report
}

\author{
Pietro Bertoglio ${ }^{*}$, Ivan Lomangino ${ }^{1,2}$, Giulia Querzoli ${ }^{3}$, Angela Bonalumi ${ }^{4}$, Giuseppe S. Bogina ${ }^{3}$, \\ Alberto C. Terzi ${ }^{1}$ \\ ${ }^{1}$ Division of Thoracic Surgery, IRCCS Sacro Cuore-Don Calabria Hospital, Negrar (VR); ${ }^{2}$ Division of Thoracic \\ Surgery, University Hospital of Padua; ${ }^{3}$ Division of Pathological Anatomy, IRCCS Sacro Cuore-Don Calabria \\ Hospital, Negrar (VR); ${ }^{4}$ Division of Hematology, University Hospital of Verona, Italy
}

\begin{abstract}
Classic Hodgkin lymphomas are neoplasms originating from lymphoid tissue. Primary extra-nodal classic Hodgkin lymphoma (PE-cHL) of the lung is rare.

A 37-years-old Caucasian male was referred to our hospital for recurrent episodes of hemoptysis, cough and bronchitis. A computed tomography (CT) scan showed a massive left upper lung consolidation, which was positive at the positron emission tomography
\end{abstract}

Correspondence: Pietro Bertoglio, Division of Thoracic Surgery, Sacro Cuore-Don Calabria Hospital, via Sempreboni 5, 37024 Negrar (VR), Italy.

Tel. +39.0456.014732.

E-mail: pieberto@hotmail.com

Authors' contributions: PB, IL, conception of the work; PB, IL, GQ, $\mathrm{AB}, \mathrm{GSB}, \mathrm{ACT}$, acquisition and interpretation of data for the work; PB, IL, GQ, drafting the work; PB, IL, GQ, AB, GSB, ACT, critical revision of the work; PB, IL, GQ, AB, GSB, ACT, final approval; PB, IL, GQ, AB, GSB, ACT, accountability for all aspects of the work.

Conflict of interest: The authors declare no potential conflict of interest.

Funding: None.

Informed consent: Written informed consent was obtained from the patient.

Availability of data and materials: All data generated or analyzed during this study are included in this published article.

Key words: Lymphoma; pulmonary primary extra-nodal classic Hodgkin lymphoma; lung resection; haemoptysis.

Received for publication: 25 January 2021.

Accepted for publication: 7 May 2021.

${ }^{\circ}$ Copyright: the Author(s), 2021

Licensee PAGEPress, Italy

Monaldi Archives for Chest Disease 2021; 91:1781

doi: 10.4081/monaldi.2021.1781

This article is distributed under the terms of the Creative Commons Attribution Noncommercial License (by-nc 4.0) which permits any noncommercial use, distribution, and reproduction in any medium, provided the original author(s) and source are credited.
(PET) scan. After several inconclusive tests and no benefit from medical therapies, the patient underwent a left upper lobectomy; pathology report showed a classical type Hodgkin lymphoma with no lymph-nodes involvement. Four cycles of adjuvant chemotherapy were administered with no toxicity. At the last follow up 14 months after surgery, the patient is alive and free from disease.

Primary extra-nodal classical Hodgkin lymphoma of the lung is a rare entity, but it should be considered as a differential diagnosis in young patients with pulmonary consolidation even without systemic symptoms.

\section{Introduction}

Primary pulmonary lymphomas represent a rare pathologically heterogeneous group of disorders representing less than $0.5 \%$ of all primary pulmonary malignancies [1]; among them, nonHodgkin lymphomas are the most frequent, while primary extranodal classic Hodgkin lymphoma (PE-cHL) of the lung has seldom been described in literature $[1,2]$.

We report a challenging and rare case of PE-cHL arising with haemoptysis and pulmonary consolidations.

\section{Case Report}

A 37-years-old Caucasian male was referred to our hospital for recurrent episodes of hemoptysis, cough and bronchitis. The patient was a former smoker (15 pack/year) for six months, he had an occupational exposure to industrial dusts and history of recurrent bronchopneumonia. A chest X-ray and a computed tomography (CT) were performed showing an asymmetry of the rib cage due to a minor expansion of the left hemithorax and an extended consolidation of the ventral and apical segment of the left upper lobe and adenopathies in the ipsilateral lymph-node stations $4 \mathrm{~L}$ and 10L (Figure $1 \mathrm{~A}-\mathrm{C}$ ).

He was therefore hospitalized at the infectious disease department with the suspected diagnosis of tuberculosis. Nevertheless, all microbiological and molecular tests were negative; concurrently, a positron emission tomography (PET)-CT (Figure 2 A,B) was performed showing an uptake of the lung consolidation with a standard uptake value (SUV) between 8 and 14, and hypermetabolic lympho-adenopathies adjacent to the left upper lobar bronchus and the left hilum (SUV between 9 and 17). Endobronchial ultrasound (EBUS) exploration did not find any 
significant mediastinal adenopathies, but a transbronchial biopsy of the lung consolidation was performed; the pathological report suggested a possible infection or a granulomatosis with polyangiitis without signs of malignancies.

Given the persistence of the symptoms and the loss of function of the left upper lobe, after multidisciplinary discussion, the patient was sent for a left upper lobectomy. The surgical resection was performed via a total muscle sparing lateral thoracotomy and a lymphnode sampling was also carried out in stations 5 (aorto-pulmonary window), 6 (para-aortic), 7 (subcarinal), 10, 11 and 12 (hilar and intrapulmonary stations); a total number of 10 lymph-nodes were retrieved. Post-operative course was uneventful.

Surprisingly, the definitive histological report revealed a clas- sical type Hodgkin lymphoma in pulmonary parenchyma of the left upper lobe (Figure 3). At microscopic analysis, B cells were positive for CD30, weak positive for PAX5 and negative for CD45, while CD15 was positive only in the paranuclear or Golgi area. All lymph-nodes retrieved were negative. The patient was then followed up by hematology department. Despite the resection of all the macroscopic disease and a postoperative PET-CT negative for further disease sites, given the peculiar features of the disease, the patient was indicated for 4 cycles of adjuvant chemotherapy according to ABVD regimen (adriamycin, bleomycin, vinblastine, dacarbazine), with no complications or toxicity. At the last follow up 14 months after surgery, the patient is in good conditions (ECOG 0$)$ and free from disease.
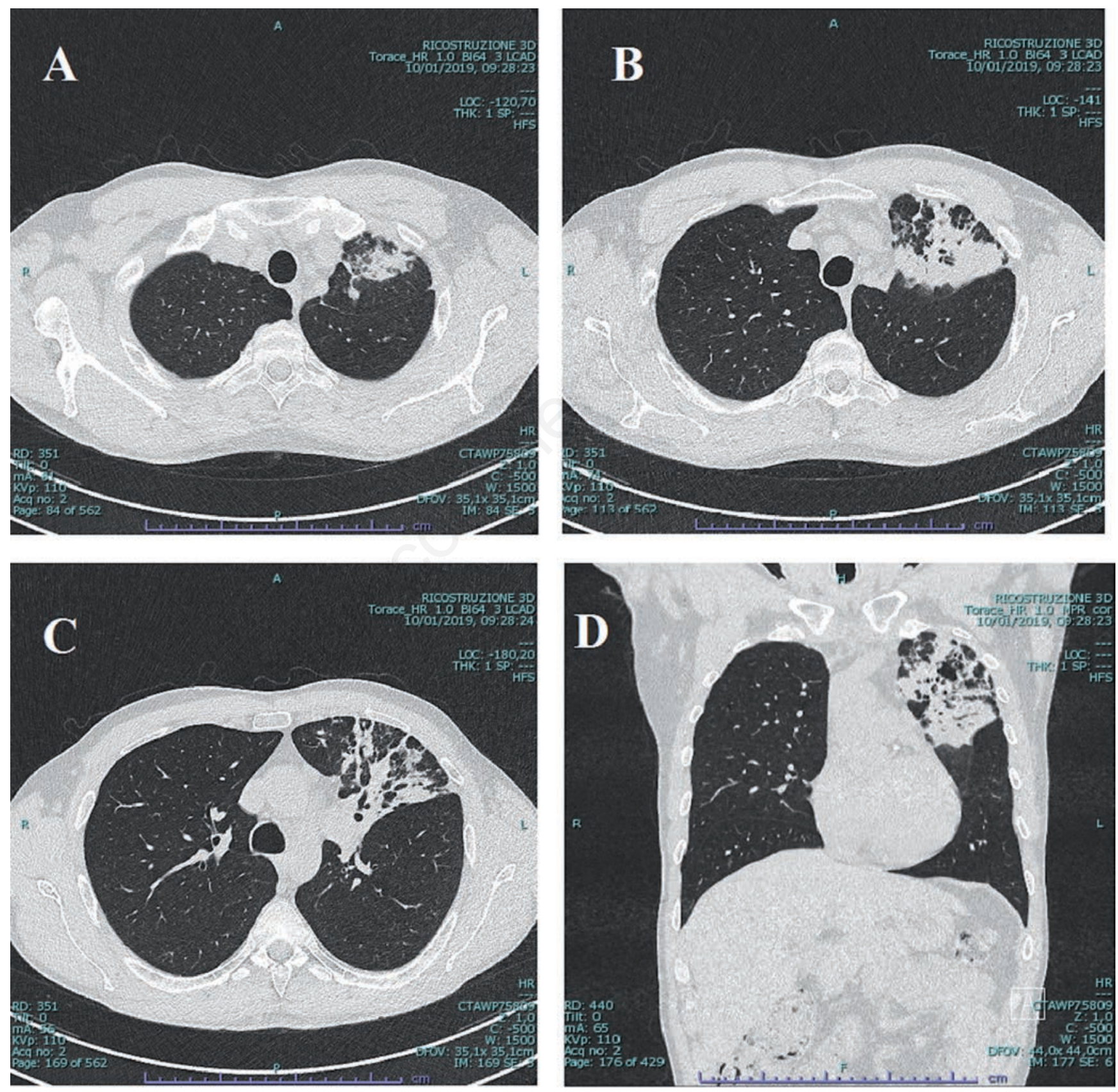

Figure 1. A,B,C) Sagittal and D) coronal images of pre-operative computed tomography scan. 


\section{Discussion}

The Lugano staging system defines PE-cHL as "diffuse or disseminated of at least one extra-nodal organ with or without associated lymph node involvement" [3]. Up to $45 \%$ of non-Hodgkin lymphomas arise from extra nodal sites, [4] and they might involve several organs such as bone, nasopharynx, parotid gland, brain and adrenal gland [5]. Lung is seldom described in literature as an extra-nodal site of development of PE-cHL and the majority of cases have been reported in young male patients located in the upper lobes.

Concurrently, the Ann Arbor classification divides lymphomas based on their spread and on to the presence (type B) or the absence (type A) of systemic symptoms (such us night sweating, fever and weight loss) [3]. In the present case, the patient had no systemic symptoms (type A) and those which were reported were only related to the lung involvement. Hemoptysis and dyspnea have been described in some case report of PE-cHL [1], but they remain non-specific symptoms which can be hardly connected with the final diagnosis. Type B symptoms have been related to a worse prognosis [3,2]; other possible negative prognostic factors are bilateral disease, multi-lobar involvement, pleural infiltration, cavitation of lung consolidation and age over 60 years. Nevertheless, data on survival of lung PE-cHL are inconsistent, but a strict follow up is mandatory to prevent recurrences $[2,5]$.

Surgery is not part of the treatment of lung PE-cHL, but it might be required to achieve a correct diagnosis as the results of fine needle biopsies can be rather controversial [2]. In the current case, lung PE-cHL was not suspected and surgery was performed as antibiotic therapies did not have benefit on symptoms and left upper lobe was almost completely involved by the disease.

In the initial assessment of this case, a transbronchial biopsy was performed with the evidence of a fibro-inflammatory tissue with lymphocytes and eosinophil granulocytes, which suggested a Wegener granulomatosis as potential differential diagnosis.
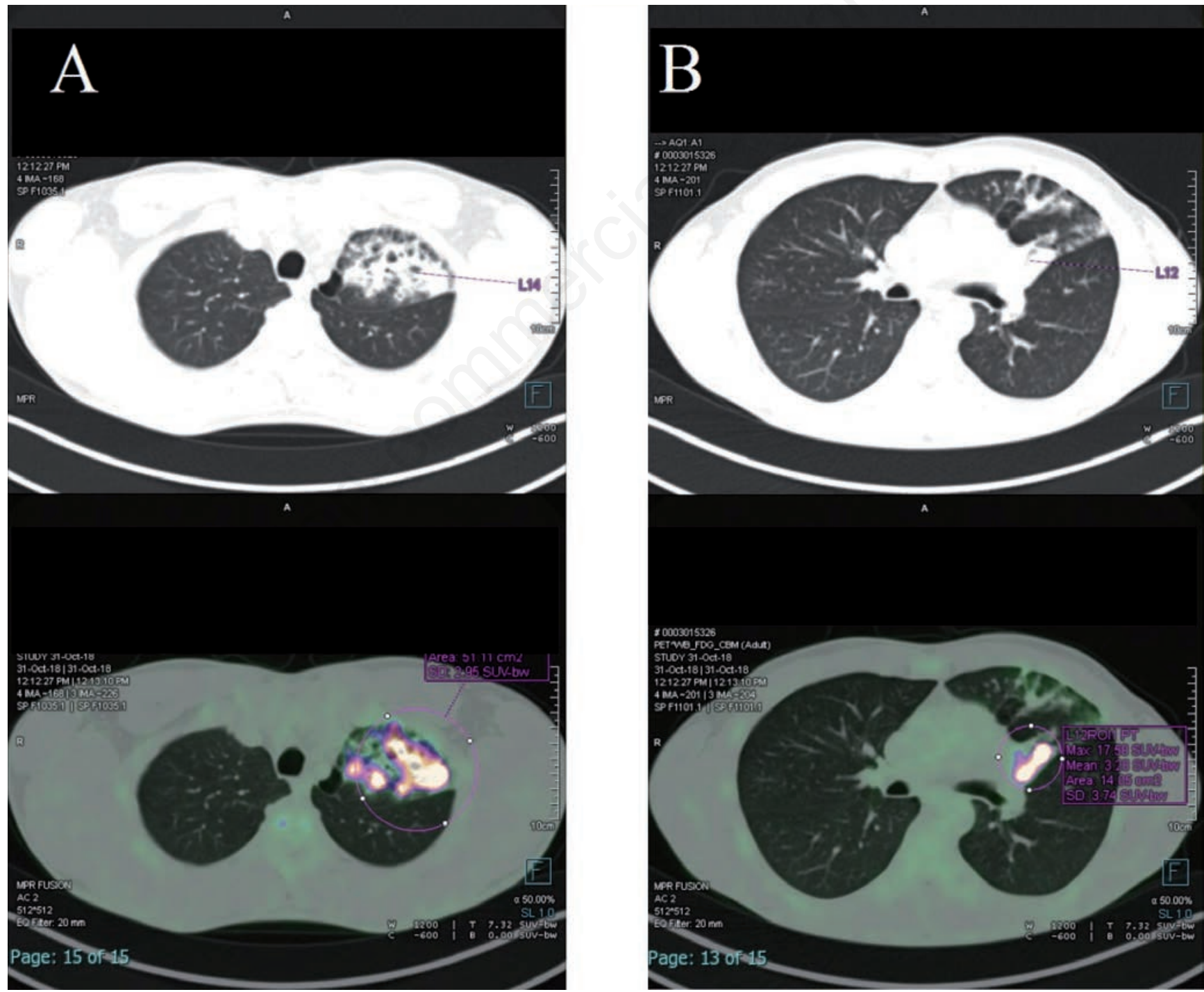

Figure 2. Pre-operative positron emission tomography/computed tomography images of A) lung consolidations and B) hilar lymph-node. 
Wegener granulomatosis has been already described as a possible differential diagnosis [1] by other authors. Diagnosis of Hodgkin lymphoma (HL) from small biopsies or citology specimen might be challenging; concurrently, the absence of clinical clues suggesting a possible lymphoproliferative disease can made the right diagnosis even harder. Kumar et al. reported an erroneous diagnosis of non-small cell lung cancer instead of Pe-CHL made with a lung fine needle aspiration citology (FNAC); the correct diagnosis was then discovered via a lymph node biopsy [6].

Microscopic analysis of lung specimen revealed the presence of a diffuse fibro-inflammatory process with lymphocytes, histiocytes and granulocytes. In this contest, pathologists found a central part rich in neutrophil granulocytes surrounded by large cells with convoluted nucleus and nucleoli with Sternbergoid features; in these cells, immunohistochemistry showed a high positivity to CD30 and a weak positivity to PAX5. Concurrently, CD15 was positive in the paranuclear or Golgi area only in part of these CD30-positive cells. Conversely, the analysis of nodal specimen did not show any positivity for CD30. The essential panel of markers including CD3, CD20, CD15, CD30 and PAX5 for the correct diagnosis of HL has been recently confirmed by a paper authored by American experts [7]; as reported in our case, positivity for CD15 is usually described as non-uniform between different cells and also inside the cells and it can be completely negative in up to $33 \%$ of classical HL. Negativity to CD45 was used to ruled out the differential diagnosis of anaplastic large cell lymphoma [8]. Moreover, in case of pulmonary specimen with microscopic features which might suggest a possible diagnosis of Pe-CHL, differential diagnosis should include Langerhans histiocytosis; for these patients, immunohistochemistry can help to exclude this possible diagnosis by the negative staining for $\mathrm{S} 100$ and CD1.

Up to $50 \%$ of classical HL in immunocompetent people are related to a previous Epstein-Barr virus (EBV) infection and it has been demonstrated to be strictly related to a worse prognosis; for this reason, assessment for presence of EBV in the specimen cells is required in case of Hodgkin lymphoma. The assessment might be done by immunohistochemistry revealing specific membrane proteins like LMP1 or by using the in situ hybridization technique to verify the presence of EBV encoded RNA inside HL cells (EBER) [9]. In our case, EBER was used and showed no signs of EBV genome in HL cells.

\section{Conclusions}

In conclusion, primary extra-nodal classical Hodgkin lymphoma of the lung is rare but it should be considered as a differential diagnosis in young patients with pulmonary consolidations even without systemic symptoms. In case of non-diagnostic workup tests or failure of pharmacological therapies, surgical biopsy (preferably using a minimally invasive technique) should be proposed to assess the correct diagnosis.
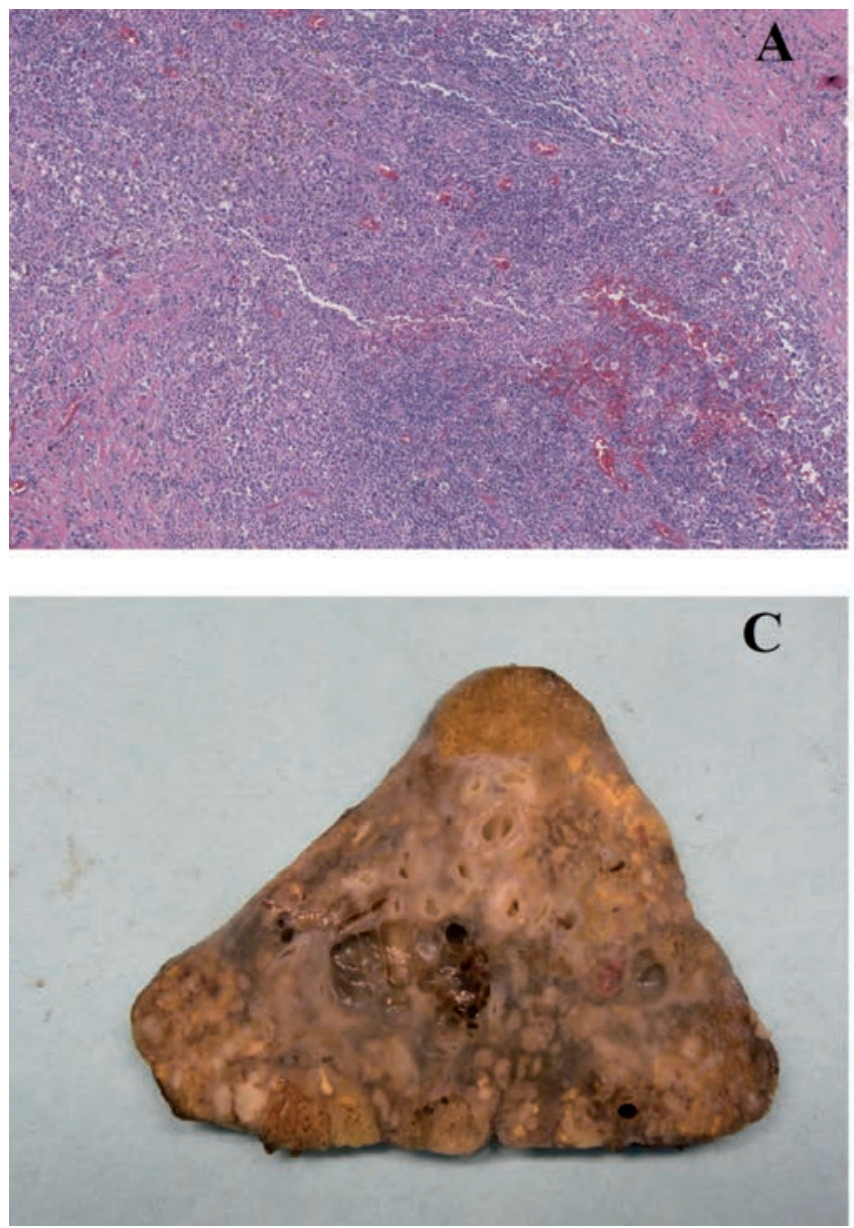

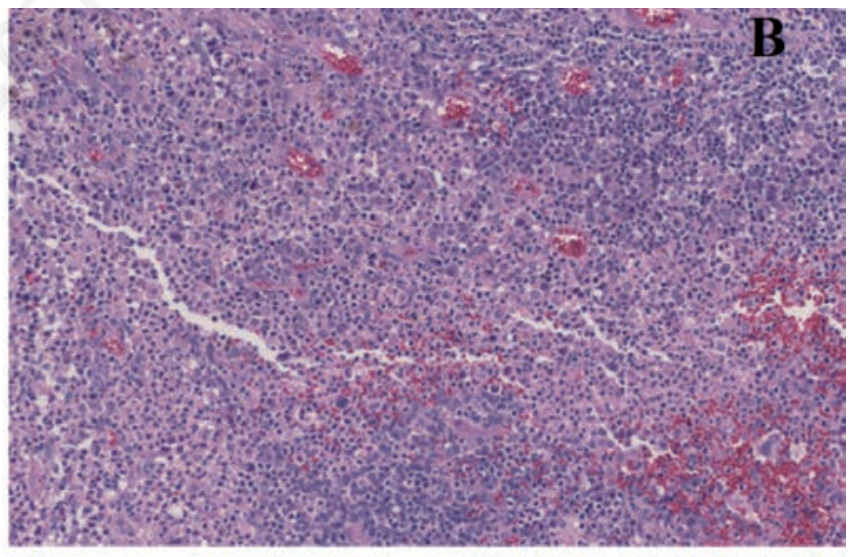

Figure 3. A,B) Microscopic slides of the specimen stained with eosin and hematoxylin, enlargement 10x $(A)$ and 20x (B) and $C)$ macroscopic view. 


\section{References}

1. Cooksley N, Judge DJ, Brown J. Primary pulmonary Hodgkin's lymphoma and a review of the literature since 2006. BMJ Case Rep 2014;2014:bcr2014204020.

2. Rodriguez J, Tirabosco R, Pizzolitto S, et al. Hodgkin lymphoma presenting with exclusive or preponderant pulmonary involvement: a clinicopathologic study of 5 new cases. Ann Diagn Pathol 2006;10:83-8.

3. Cheson BD, Fisher RI, Barrington SF, et al. Recommendations for initial evaluation, staging, and response assessment of Hodgkin and non-Hodgkin lymphoma: the Lugano classification. J Clin Oncol 2014;32:3059-68.

4. Cavalli F, Stein H, Zucca E. Extranodal lymphomas pathology and management. Informa: London; 2008; 331 pp.
5. Yang M, Ping L, Liu W, et al. Clinical characteristics and prognostic factors of primary extranodal classical Hodgkin lymphoma: a retrospective study. Hematology 2019;24:413-9.

6. Kumar R, Sidhu H, Mistry R, Shet T. Primary pulmonary Hodgkin's lymphoma: a rare pitfall in transthoracic fine needle aspiration cytology. Diagn Cytopathol 2008;36:666-9.

7. O'Malley DP, Dogan A, Fedoriw Y, et al. American Registry of Pathology Expert Opinions: immunohistochemical evaluation of classic Hodgkin lymphoma. Ann Diagn Pathol 2019;39:105-10

8. Muzzafar T, Wei EX, Lin P, et al. Flow cytometric immunophenotyping of anaplastic large cell lymphoma. Arch Pathol Lab Med 2009;133:49-56.

9. Gulley ML, Glaser SL, Craig FE, et al. Guidelines for interpreting EBER in situ hybridization and LMP1 immunohistochemical tests for detecting Epstein-Barr virus in Hodgkin lymphoma. Am J Clin Pathol 2002;117:259-67. 